Classification

Physics Abstracts

$61.10-61.50 \mathrm{~K}$

\title{
Etude de la structure de la phase haute température de la triéthylènediamine sur des monocristaux fabriqués à $388 \mathrm{~K}$
}

\author{
A. Hedoux, J. L. Sauvajol ${ }^{+}$, J. Lefebvre et M. Muller \\ Laboratoire de Dynamique des Cristaux Moléculaires, UA 801, \\ Bât. P5, Université des Sciences et Techniques de Lille, 59655 Villeneuve d'Ascq Cedex, France
}

(Reçu le 26 novembre 1985, révisé le 20 janvier 1986, accepté le 24 janvier 1986)

\begin{abstract}
Résumé. - Le triéthylènediamine $\left(\mathrm{N}\left[\mathrm{CH}_{2} \mathrm{CH}_{2}\right]_{3} \mathrm{~N}\right)$ présente une transition de phase solide ordonné-solide plastique à $T_{\mathrm{c}}=351 \mathrm{~K}$. Des monocristaux de triéthylènediamine dans sa phase haute température ont été obtenus in situ sur le diffractomètre par la méthode de Bridgmann. La structure a été déterminée à $T=388 \mathrm{~K}$. Deux modèles ont été utilisés pour résoudre la structure; le premier est un modèle de désordre statique, le second consiste à développer sur une base de fonctions adaptées à la symétrie du site et de la molécule le facteur de structure. Les résultats confirment le groupe spatial $\mathrm{Fm} 3 \mathrm{~m}$ ainsi que l'orientation de l'axe d'ordre trois moléculaire le long de la direction $\langle 111\rangle$ du cube avec deux positions d'équilibre.
\end{abstract}

\begin{abstract}
Triethylenediamine $\left(\mathrm{N}^{2}\left[\mathrm{CH}_{2} \mathrm{CH}_{2}\right]_{3} \mathrm{~N}\right)$ undergoes a solid state structural transition at $351 \mathrm{~K}$. Single crystals of phase I (above $351 \mathrm{~K}$ ) have been grown in situ on the diffractometer by the Bridgmann method. The structure has been determined at $T=388 \mathrm{~K}$. To solve the structure, two refinement procedures have been used : Frenkel model and decomposition of the orientational average density of the atoms on symmetry-adapted functions. The results confirm the space group $\mathrm{Fm} 3 \mathrm{~m}$ and coincidence of the three fold molecular and crystal axes. Around each $\langle 111\rangle$ direction there are two orientations separated by a $60^{\circ}$ rotation about that direction.
\end{abstract}

\section{Introduction.}

La triéthylènediamine(TEDA, 1,4-diazabicyclo $[2,2,2]$ octane, $\left.\mathrm{N}\left(\mathrm{CH}_{2} \mathrm{CH}_{2}\right)_{3} \mathrm{~N}\right)$ (Fig. 1) présente une transition du type ordre-désordre à $T_{\mathrm{c}}=351 \mathrm{~K}$ [1].

La structure cristallographique de la phase II ordonnée a fait l'objet de plusieurs déterminations $[2,3]$. Elle est hexagonale compacte (hcp) de groupe spatial $\mathrm{P}_{3} / \mathrm{m}(Z=2)$. Une analyse de la variation avec la température des coefficients d'agitation thermique de libration [3] montre un accroissement de l'anisotropie de ce tenseur avec la température, ceci à cause d'une forte croissance de l'amplitude de libration $\left(L_{33}\right)^{1 / 2}$ autour de l'axe moléculaire d'ordre trois. Ce résultat est en accord avec des données de résonance magnétique nucléaire [4] qui décrivent la phase II comme un réseau "rigide " en dessous de $T=190 \mathrm{~K}$ et " anharmonique » au-dessus.

Une première détermination de la structure cristallographique de la phase haute température (phase I)

$\left({ }^{+}\right)$Adresse actuelle : Laboratoire d'Infrarouge (G.D.P.C.), Place Eugène Bataillon, 34060 Montpellier Cedex, France.

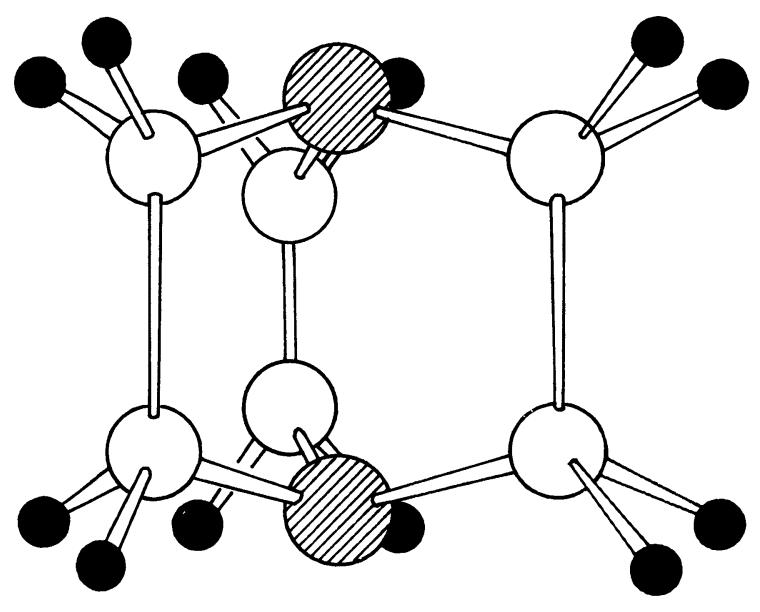

Fig. 1. - La molécule de triéthylènediamine de symétrie $D_{3 h}$.

[Triethylenediamine molecule-molecular symmetry $\mathrm{D}_{3 \mathrm{~h}}$.]

de TEDA a été réalisée à partir de données de diffraction $X$ sur poudre par Nimmo et Lucas [5]. Onze raies de diffraction d'intensité significative ont été miesurées 
et les deux raies les plus intenses du spectre (200) et (111) ont été éliminées dans leurs affinements à cause des possibilités d'extinction ce qui laisse neuf valeurs d'intensité pour déterminer la structure de cette phase. Dans cette étude, il a été montré que le groupe spatial est $\mathrm{Fm} 3 \mathrm{~m}(Z=4)$, que les axes d'ordre trois moléculaires s'alignent en moyenne le long des directions $\langle 111\rangle$ du cube. Autour de chaque direction $\langle 111\rangle$ la molécule possède deux orientations d'équilibre séparées de $60^{\circ}$ (Fig. 2), le plan de symétrie moléculaire $\sigma_{\mathrm{v}}$ faisant un angle de $30^{\circ}$ avec la direction $\mathbf{a}+\mathbf{b}-2 \mathbf{c}$.

Ayant pour objectif l'étude des mouvements moléculaires de TEDA dans ses différentes phases, il nous a paru nécessaire de reprendre la détermination de la structure de la phase I de TEDA à partir de données de diffraction $X$ sur monocristal afin d'affiner et de compléter les résultats de Nimmo et Lucas [5].

\section{Fabrication d'un monocristal de la phase I de TEDA A $T=388 \mathrm{~K}$.}

La transition solide ordonné-plastique à $T_{\mathrm{c}}$ étant du premier ordre et fortement destructive, le cristal de la phase I de TEDA a été fabriqué in situ sur le diffractomètre automatique à $T=388 \mathrm{~K}$. La méthode employée s'apparente à la technique de Bridgmann. Une très faible quantité de poudre de TEDA purifiée par sublimations successives et sur tamis moléculaire est introduite dans un capillaire de Lindemann de diamètre $\phi=0,3 \mathrm{~mm}$. Ce tube est placé dans une gaine de laiton, lui-même fixé dans un cylindre de cuivre mis en température par une résistance dont le chauffage peut être régulé pour obtenir au niveau de l'échantillon une température fixe(Fig. 3). Toute la poudre de TEDA est dans un premier temps liquéfiée $\left(T_{\mathrm{m}}=433 \mathrm{~K}\right)$. La température est ensuite abaissée très lentement jusqu'à l'observation d'un début de cristallisation.

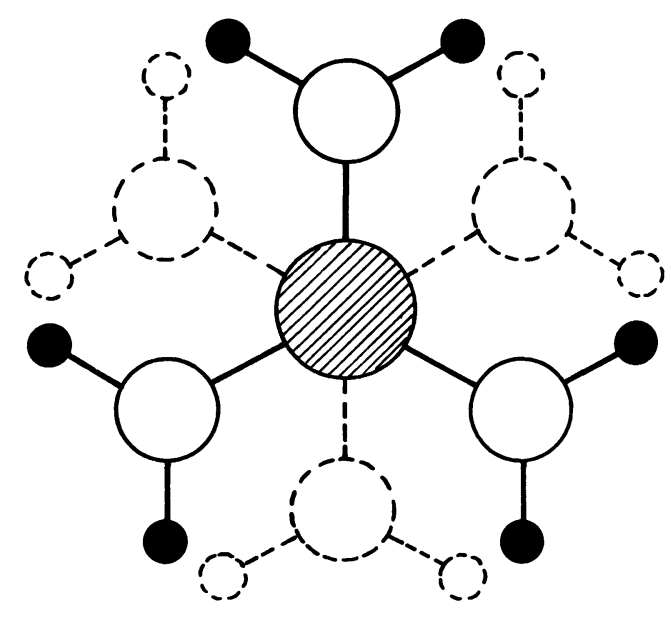

Fig. 2. - Les deux orientations possibles de la molécule autour de l'axe $\langle 111\rangle$ du cube séparées d'un angle de $60^{\circ}$.

[The two possible orientations of the molecule around each $\langle 111\rangle$ direction, separated by a $60^{\circ}$ rotation about that direction.]

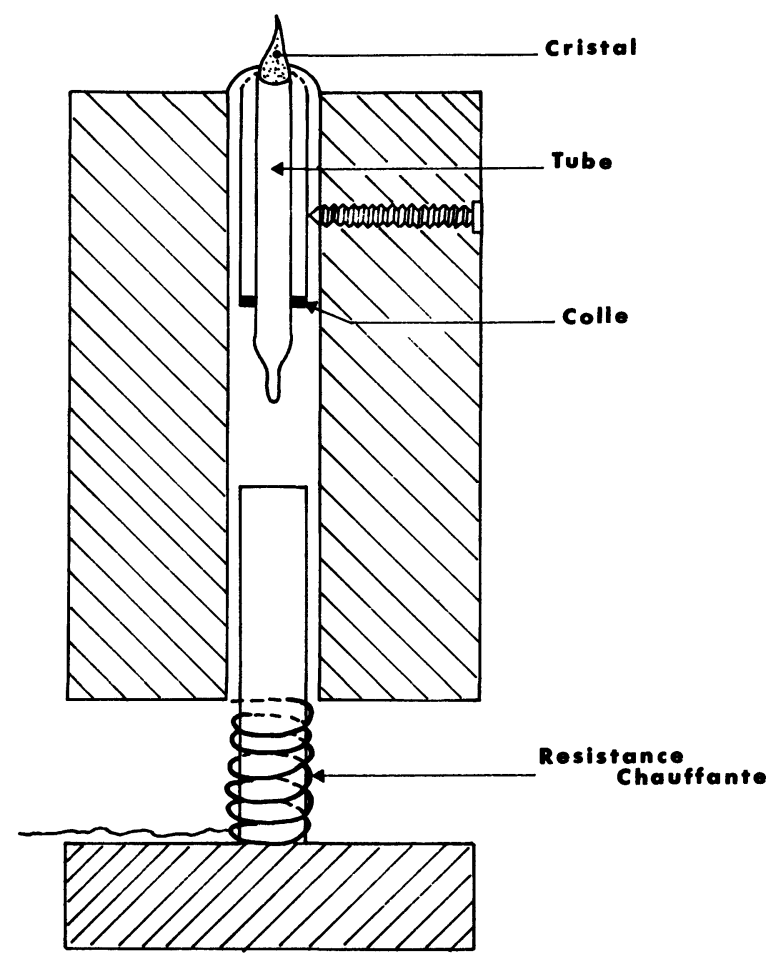

Fig. 3. - Coupe du four utilisé pour fabriquer le monocristal TEDA.

[Section of the furnace used to grow the single crystals of TEDA.]

Plusieurs cycles de température sont alors effectués autour du point où cette cristallisation est apparue. Ensuite la température est de nouveau abaissée lentement et la progression de la cristallisation est contrôlée par la modification de l'interface liquidesolide. Cette méthode s'est révélée capable de fournir des germes monocristallins de la phase I de TEDA. La qualité de ceux-ci a été testée à partir du profil des raies de diffraction qui ont une finesse typique d'un pic de Bragg et qui ne présentent aucune disymétrie. De plus la recherche systématique des raies de diffraction par le programme de « Peak Hunting » du diffractomètre Philips PW 1100 fournit un jeu de données qui s'indexent à l'aide d'un seul jeu de paramètres $(h, k, l)$ confirmant ainsi le caractère monocristallin des germes utilisés.

\section{Obtention et corrections des intensités diffractées.}

Les données de diffraction $\mathrm{X}$ ont été enregistrées sur un diffractomètre automatique 4 cercles Philips PW 1100. La radiation incidente est la raie $K_{\alpha}$ du molybdène $(\lambda=0,7107 \AA)$. Les intensités intégrées des raies de diffraction ont été obtenues par un balayage $\theta-2 \theta$ sur une largeur de $2^{\circ}$ en $\theta$ à la vitesse de 0,6 degré par minute. Le domaine angulaire en $\theta$ a été fixé de $3^{\circ}$ à $25^{\circ}$, d'autre part compte tenu de l'encombrement du four l'angle azimutal de balayage $\chi$ a été limité à $40^{\circ}$. Les données ont été corrigées du facteur de 
Lorentz-polarisation. Aucune correction d'absorption n'a été réalisée.

Finalement 71 réflexions ont été mesurées correspondant à 19 raies non équivalentes ayant une intensité significative $\left(I_{\text {obs }}>3 \sigma\right)$ (Tableau I). Elles s'indexent dans un réseau cubique de paramètre $a=8,89 \AA$ à $T=388 \mathrm{~K}$. Les 19 réflexions mesurées ont les extinctions systématiques

et

$$
h+k=2 n+1, k+1=2 n+1
$$

$$
l+h=2 n+1
$$

ce qui permet de conclure en un réseau cubique à faces centrées.

De plus, l'égalité des intensités

$$
I(h, k, l)=I(\bar{k}, h, l)
$$

donne comme possibilités l'un des trois groupes spatiaux suivants :

$$
\mathrm{Fm} 3 \mathrm{~m}, \overline{\mathrm{F}} \overline{4} \mathrm{~m} \mathrm{~m} \text { et } \mathrm{F} 432 \text {. }
$$

Tableau I. - Facteurs de structure observés et facteurs de structure calculés pour les deux modèles utilisés. Les raies précédées d'un astérisque sont celles utilisées par Nimmo et Lucas pour résoudre la structure.

[The observed structure factors and the corresponding

\begin{tabular}{|c|c|c|c|}
\hline$(h, k, l)$ & $\begin{array}{l}\text { Fact. } \\
\text { obs. }\end{array}$ & $\begin{array}{c}\text { Fact. calc. } \\
\text { (F.A.S.) }\end{array}$ & $\begin{array}{c}\text { Fact. calc. } \\
\text { (T.L.S.) }\end{array}$ \\
\hline * 1111 & 1536,31 & 2069,25 & 1477,76 \\
\hline * 200 & 1194,85 & 1421,70 & 999,31 \\
\hline * 222 & 22,75 & 27,70 & 59,72 \\
\hline * 311 & 220,57 & 217,13 & 228,34 \\
\hline * 222 & 371,60 & 325,87 & 363,94 \\
\hline * 400 & 229,05 & 228,20 & 226,78 \\
\hline * 331 & 196,97 & 190,75 & 193,91 \\
\hline * 420 & 148,95 & 159,97 & 143,56 \\
\hline * 333 & 139,03 & 135,08 & 150,82 \\
\hline * 5111 & 101,52 & 107,03 & 98,13 \\
\hline 440 & 45,15 & 46,30 & 43,09 \\
\hline * 422 & 146,27 & 134,17 & 133,72 \\
\hline 531 & 18,56 & 17,32 & 25,06 \\
\hline 600 & 67,77 & 71,06 & 74,94 \\
\hline 551 & 50,57 & 50,82 & 71,66 \\
\hline 711 & 16,27 & 12,29 & 11,48 \\
\hline 640 & 47,68 & 46,24 & 61,36 \\
\hline 462 & 29,47 & 19,75 & 38,17 \\
\hline \multirow[t]{2}{*}{713} & 18,59 & 12,53 & 18,74 \\
\hline & $\begin{array}{c}R \\
W R\end{array}$ & $\begin{array}{l}7,65 \times 10^{-2} \\
7,24 \times 10^{-2}\end{array}$ & $\begin{array}{l}6,21 \times 10^{-2} \\
3,45 \times 10^{-2}\end{array}$ \\
\hline
\end{tabular}
calculated data for the two models described in the text. The lines marked by an asterisk have been used by Nimmo and Lucas to determine the structure.]
La procédure d'affinement utilisée consiste à comparer les facteurs de structure observés et calculés reliés par la relation :

$$
\left|F_{\text {obs }}\right|=a\left|F_{\text {cal }}\right|
$$

où $a$ est un facteur d'échelle (qui est un paramètre dans l'affinement).

Un affinement par moindres carrés consistant à minimiser l'expression :

$$
\sum_{i=1}^{N} W_{i}\left(\left|F_{\mathrm{obs}}^{i}\right|-a\left|F_{\mathrm{cal}}^{i}\right|\right)^{2}
$$

a été employé. Dans le terme précédent, la somme porte sur le nombre $N$ d'intensités mesurées ; $W_{i}$ est un poids statique affecté à chaque intensité mesurée. La convergence de l'affinement est testée à partir de deux facteurs de mérite :

$$
R=\sum_{i=1}^{N}|| F_{\mathrm{obs}}^{i}|-a| F_{\mathrm{cal}}^{i} \| / \sum_{i=1}^{N}\left|F_{\mathrm{obs}}^{i}\right|
$$

$R_{\mathrm{w}}=\left[\sum_{i=1}^{N} W_{i}\left(\left|F_{\mathrm{obs}}^{i}\right|-a\left|F_{\mathrm{cal}}^{i}\right|\right)^{2} / \sum_{i=1}^{N} W_{i}\left(\left|F_{\mathrm{obs}}^{i}\right|\right)^{2}\right]^{1 / 2}$

$W_{i}$ est pris égal à l'inverse du carré de l'écart type.

Dans la procédure d'affinement les deux raies intenses du spectre (200) et (111) dont les intensités peuvent être de ce fait affaiblies par les extinctions secondaires ont été omises. Les facteurs de structure correspondants ont été calculés a posteriori à partir des données de l'affinement sur les dix-sept raies restantes.

La structure à résoudre étant celle d'une phase à désordre orientationnel, deux approximations majeures ont été prises en compte :

a) Les molécules sont considérées comme rigides, et ainsi des procédures d'affinement en blocs rigides ont été utilisées. En particulier des facteurs d'agitation thermique moléculaire sont introduits sans passer par l'intermédiaire des coefficients thermiques de chaque atome.

b) La configuration de la molécule (symétrie et distances interatomiques) est identique à celle déterminée par l'affinement des positions atomiques à basse température.

Le facteur de structure peut s'écrire dans ce cas sous la forme :

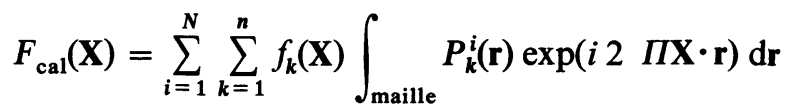

où

$f_{k}(\mathbf{X})$ est le facteur de diffusion atomique de l'atome $k$ $\mathbf{X}$ le vecteur de diffusion

$P_{k}^{i}(\mathbf{r})$ la probabilité de trouver l'atome $i$ de la molécule $k$ en $\mathbf{r}$

$N$ le nombre de molécules dans la maille $n$ le nombre d'atomes par molécule. 
Dans la phase plastique $P_{k}^{i}(r)$ doit rendre compte non seulement de l'agitation thermique mais aussi du désordre d'orientation. Deux modèles ont été utilisés pour prendre en compte le désordre orientationnel.

a) Un modèle de désordre statique qui consiste à introduire pour chaque maille le nombre $D$ de molécules par site, nécessaire pour rendre compte de la symétrie moyenne en affectant un poids $1 / D$ à chaque molécule. Le programme Orion [6] permet de décrire cette situation.

b) Un modèle qui consiste à développer sur une base de fonctions adaptées à la symétrie du site et de la molécule le facteur de structure. On définira une probabilité d'orientation $P_{0}(\Omega)$ par : [7]

$$
P_{0}(\Omega)=\frac{1}{8 \Pi^{2}} \sum_{\left(\begin{array}{c}
l \\
\lambda \lambda^{\prime}
\end{array}\right)} A_{l}^{\lambda \lambda^{\prime}}(2 l+1) U_{l}^{\lambda \lambda^{\prime}}(\Omega)
$$

où les $U_{l}^{\lambda \lambda^{\prime}}(\Omega)$ sont les fonctions adaptées à la symétrie (d'ordre $l$ ); $\lambda, \lambda^{\prime}$ indexant respectivement les représentations irréductibles totalement symétriques du groupe du site et de la molécule. Les coefficients $A_{l}^{\lambda \lambda^{\prime}}$ sont des paramètres dans la procédure d'affinement. $\Omega$ représente les angles qui repèrent l'orientation du trièdre moléculaire par rapport au trièdre cristallographique.

\section{Analyse des résultats.}

4.1 Désordre STATIQUE. - Les valeurs de départ des paramètres sont les valeurs obtenues par Nimmo et Lucas [2] (Tableau II). L'axe d'ordre trois moléculaire est aligné suivant les directions $\langle 111\rangle \mathrm{du}$ système cubique. Dans ce cas le groupe minimum de symétrie du site est $\mathrm{C}_{3}$ et le tenseur TLS à la forme suivante dans un système lié à la symétrie $\left(\mathrm{O}^{\prime}\right.$ est pris parallèle à l'axe $\mathrm{C}_{3}$ moléculaire).

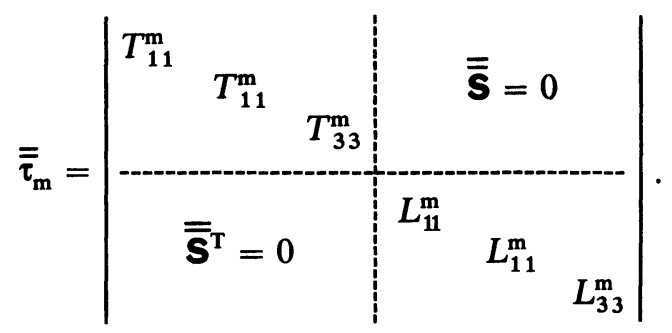

Nous avons imposé au tenseur $\overline{\overline{\mathbf{S}}}$ d'être nul dans la mesure où le petit nombre de raies significatives enregistrées ne permet pas de définir tous les paramètres et que généralement dans une phase à désordre orientationnel l'importance de ces termes n'intervient que peu dans la définition des positions d'équilibre moléculaires. Par contre l'importance et la symétrie de ces coefficients peuvent donner des renseignements intéressants, quant au processus qui pilote la transition de phase dans la mesure où le couplage translationrotation est généralement concerné dans une transition ordre-désordre.
Tableau II. - Coordonnées réduites des atomes introduits dans la procédure d'affinement exprimées dans un repère orthogonal tel que l'axe $\mathrm{O} z^{\prime}$ est aligné suivant la direction 〈111〉 du cube.

[Fractional coordinates used in the refinement procedure. They are given in the orthogonal axis system. $\mathrm{O} z^{\prime}$ is along the $\langle 111\rangle$ direction.]

\begin{tabular}{lrrr} 
Atomes & $x$ & \multicolumn{1}{c}{$y$} & \multicolumn{1}{c}{$z$} \\
C & - & \multicolumn{1}{c}{-} & - \\
C & 0,159 & $-0,060$ & 0,050 \\
H (1) & 0,262 & 0,003 & $-0,003$ \\
H (2) & 0,184 & $-0,112$ & 0,153 \\
N & 0,083 & 0,083 & 0,083
\end{tabular}

Ce même tenseur exprimé dans le repère cristallographique du système cubique à faces centrées est :

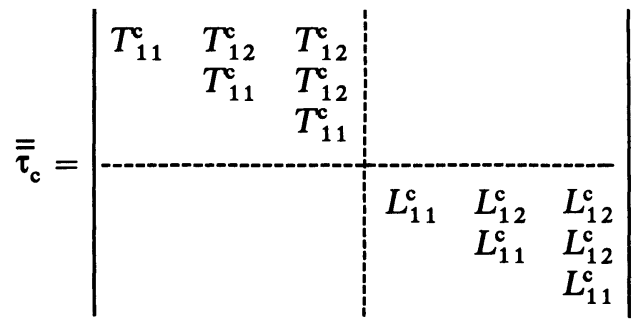

avec

$$
\begin{array}{lll}
T_{11}^{\mathrm{m}}=T_{11}^{\mathrm{c}}-T_{12}^{\mathrm{c}} & T_{33}^{\mathrm{m}}=T_{11}^{\mathrm{c}}+2 T_{12}^{\mathrm{c}} \\
L_{11}^{\mathrm{m}}=L_{11}^{\mathrm{c}}-L_{12}^{\mathrm{c}} \text { et } \quad L_{33}^{\mathrm{m}}=L_{11}^{\mathrm{c}}+2 L_{12}^{\mathrm{c}} .
\end{array}
$$

Le programme Orion a été utilisé comme procédure d'affinement [6] avec les conditions suivantes :

- Les huit positions d'équilibre de la molécule obtenues par Nimmo et Lucas (Fig. 2) ont été fixées, chaque orientation ayant une probabilité $1 / 8$.

- Le programme Orion permet de déterminer les termes du tenseur $\overline{\bar{\tau}}_{\mathrm{m}}$.

- L'angle $\alpha$ qui décrit l'orientation du plan $\sigma_{v}$ moléculaire par rapport à la direction $\langle 11 \overline{2}\rangle$ (Fig. 4) est un paramètre de l'affinement.

Les résultats sont résumés dans le tableau III.

La détermination du groupe spatial a été effectuée à partir des trois groupes sélectionnés précédemment. L'hypothèse F432 est éliminée, compte tenu que ce groupe ne possède aucun plan de symétrie. De plus, l'existence de huit positions d'équilibre élimine la solution $\mathrm{F} \overline{4} 3 \mathrm{~m}$ et impose uniquement $\mathrm{Fm} 3 \mathrm{~m}$ comme groupe spatial.

Les axes d'ordre trois moléculaires sont alignés le long des directions $\langle 111\rangle$ du cube. Autour de chaque direction $\langle 111\rangle$ la molécule possède deux orientations d'équilibre. La différence essentielle avec les résultats de Nimmo et Lucas concerne l'orientation du plan moléculaire $\sigma_{\mathrm{v}}$. Ce dernier est confondu avec le plan diagonal du réseau cubique $\left(\alpha=0^{\circ}\right)$ dans notre affinement, contrairement aux résultats de Nimmo et Lucas $\left(\alpha=30^{\circ}\right)$. 
Tableau III. - Paramètres structuraux de la triéthylènediamine $(\grave{a} T=388 \mathrm{~K})$ relatifs au modèle de désordre statique.

[Structural parameters of triethylenediamine (at $T=388 \mathrm{~K}$ ) corresponding to the Frenkel model.]

\begin{tabular}{lccccccc} 
& $T_{11}\left(\AA^{2}\right)$ & $T_{12}\left(\AA^{2}\right)$ & $T_{33}\left(\AA^{2}\right)$ & $L_{11}\left(\mathrm{rd}^{2}\right)$ & $L_{12}\left(\mathrm{rd}^{2}\right)$ & $L_{33}\left(\mathrm{rd}^{2}\right)$ & $R, W R$ \\
\cline { 2 - 7 } $\begin{array}{c}\text { Repère } \\
\text { cristal. }\end{array}$ & $\begin{array}{c}0,127 \\
(0,02)\end{array}$ & $\begin{array}{c}-0,035 \\
(0,009)\end{array}$ & $\begin{array}{c}0,127 \\
(0,02)\end{array}$ & $\begin{array}{c}0,045 \\
(0,005)\end{array}$ & 0 & $\begin{array}{c}0,045 \\
(0,005)\end{array}$ & $R=6,21 \times 10^{-2}$ \\
\hline $\begin{array}{c}\text { Repère } \\
\text { mol. }\end{array}$ & 0,162 & - & 0,052 & 0,045 & - & 0,045 & $W R=3,45 \times 10^{-2}$
\end{tabular}

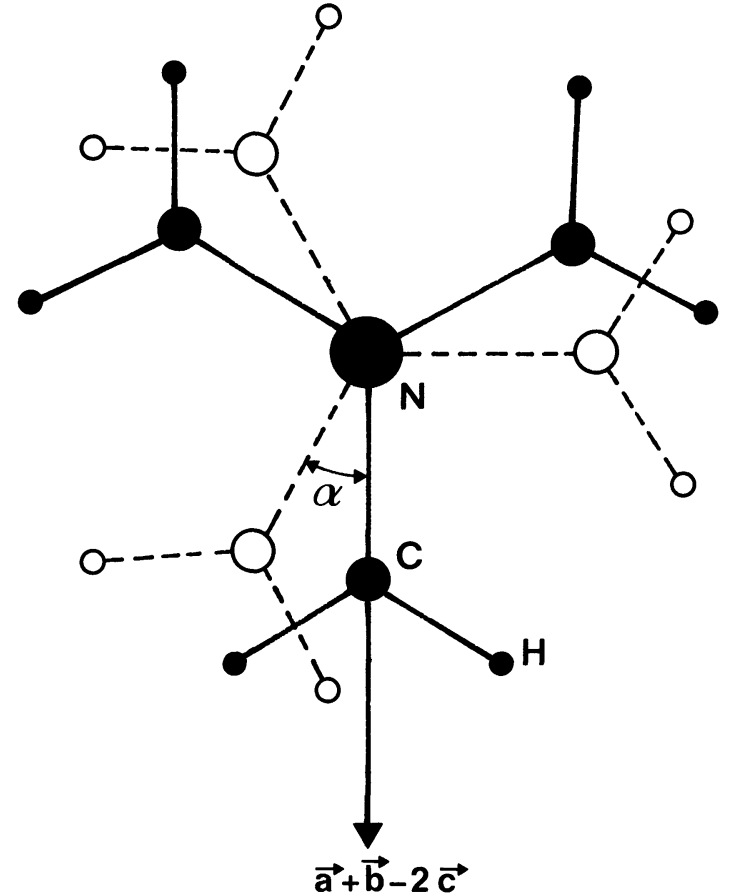

Fig. 4. - Position d'équilibre de la molécule de TEDA autour de la direction $\langle 111\rangle$ du cube. Les atomes représentés en blanc correspondent à la position de Nimmo et Lucas $\left(\alpha=30^{\circ}\right)$ et les atomes représentés en noir à la position calculée dans ce travail $\left(\alpha=0^{\circ}\right)$.

[Equilibrium position of the TEDA molecule around $\langle 111\rangle$ direction. White atoms correspond to the position of Nimmo and Lucas $\left(\alpha=30^{\circ}\right)$ and black atoms to the position of the present work $\left(\alpha=0^{\circ}\right)$.]

Les facteurs d'agitation thermique de translation expriment une amplitude de translation plus forte le long des directions perpendiculaires à la direction $\langle 111\rangle$ que suivant cette direction.

$$
\begin{array}{ll}
T_{11}^{\mathrm{m}}=0,16(2) \AA^{2} & \left(T_{11}^{\mathrm{m}}\right)^{1 / 2} \# 0,4 \AA \\
T_{33}^{\mathrm{m}}=0,05(2) \AA^{2} & \left(T_{33}^{\mathrm{m}}\right)^{1 / 2} \# 0,2 \AA .
\end{array}
$$

4.2 DÉVELOPPEMENT DU FACTEUR DE STRUCTURE EN FONCTIONS ADAPTÉES À LA SYMÉTRIE. - Dans ce modèle le désordre d'orientation est pris en compte par une fonction de probabilité d'orientation $P_{0}(\Omega)$.
Ce formalisme est plus général en théorie que le précédent puisqu'il permet de décrire toutes les situations d'un désordre isotrope $\left(P_{0}(\Omega)=1\right)$ jusqu'à un désordre de type Frenkel $P_{0}(\Omega)=\frac{1}{D} \sum_{i} \delta\left(\Omega-\Omega^{i}\right)$ où $\Omega^{i}$ décrit l'orientation d'équilibre de la molécule. Toutefois, son application effective est limitée par certaines contraintes qu'on peut analyser à partir de l'expression suivante du facteur de structure exprimé dans le formalisme [7].

où

$$
F_{\text {cal }}(\mathbf{X})=\exp \left(-2 \Pi^{2} X^{2}\left\langle u_{x}^{2}\right\rangle\right) F_{\text {rot }}(\mathbf{X})
$$

$F_{\text {rot }}(\mathbf{X})=$

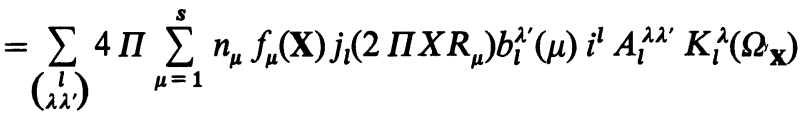
où

- $j_{l}$ est la fonction de Bessel sphérique d'ordre $l$

- $K_{l}^{\lambda}\left(\Omega_{\mathbf{X}}\right)$ les fonctions adaptées à la symétrie du site, dans notre cas ce sont les harmoniques cubiques [8]

- $\mu$ les différentes couches atomiques de la molécule au nombre de $s$

- $n_{\mu}$ le nombre d'atomes de la couche $\mu$

- $R_{\mu}$ la distance d'un atome de la couche $\mu$ au centre de masse

- $b_{l}^{\lambda^{\prime}}(\mu)$ sont des facteurs de forme moléculaire donnés par la relation

$$
b_{l}^{\lambda^{\prime}}(\mu)=M_{l}^{\lambda^{\prime}}\left(\Omega_{\mu}\right)
$$

- $M_{l}^{\lambda^{\prime}}\left(\Omega_{\mu}\right)$ est la fonction adaptée à la symétrie moléculaire et $\Omega_{\mu}$ repère la position d'équilibre d'un atome par rapport au trièdre moléculaire.

On rappelle que dans la sommation n'apparaît que les représentations irréductibles totalement symétriques du groupe du site et de la molécule.

Les limitations d'utilisation sont de trois ordres :

- Si la molécule est fortement localisée en orientation, le nombre de coefficients $A_{l}^{\lambda \lambda^{\prime}}$ est trop important pour pouvoir être affiné correctement.

- Si la taille de la molécule est importante $\left(R_{\mu}\right.$ grand) la dépendance de $j_{l}$ avec $R_{\mu}$ oblige à développer 
l'expression précédente jusqu'à des ordres en $l$ élevés; on retrouve alors la difficulté énoncée précédemment.

- Enfin quand l'ordre $l$ du développement augmente, le nombre de représentations totalement symétriques $n\left(\lambda^{\prime}, l\right)$ peut augmenter et dans le cas où $n_{\mu}<n\left(\lambda^{\prime}, l\right)$ les paramètres $b_{l}^{\lambda^{\prime}}(\mu)$ ne peuvent plus être déterminés d'une manière unique.

Pour toutes ces raisons l'application de ce formalisme est facilitée pour des molécules de petite taille possédant un nombre important de couches atomiques, avec un désordre d'orientation important.

$\mathrm{La}$ triéthylènediamine rentre par différents aspects dans cette catégorie.

Nous avons montré récemment [7] que dans le cas d'une symétrie moléculaire $\mathrm{D}_{3 \mathrm{~h}}$, la probabilité d'orientation $P_{0}(\Omega)$ pouvait se décomposer en deux séries :

$$
\begin{aligned}
P_{0}(\Omega)=\frac{1}{8 \Pi^{2}} \sum_{l} A_{l}^{11}(2 l+1) U_{l}^{11}(\Omega)+ \\
+\frac{1}{8 \Pi^{2}} \sum_{\substack{l=6 \\
\left(\lambda, \lambda^{\prime} \neq 1\right)}} A_{l}^{\lambda \lambda^{\prime}}(2 l+1) U_{l}^{\lambda \lambda^{\prime}}(\Omega) .
\end{aligned}
$$

La première série rend compte de l'orientation de l'axe d'ordre trois moléculaire, la seconde prend en compte les orientations uniaxiales.

Par notre procédure d'affinement nous pouvons donc déterminer :

- le facteur de température $\left\langle U_{x}^{2}\right\rangle$

- les coefficients $A_{l}^{\lambda \lambda^{\prime}}$.

Les résultats correspondants sont indiqués dans le tableau IV.

La probabilité d'orientation $P_{0}(\Omega)$ calculée à partir des coefficients $A_{l}^{\lambda \lambda^{\prime}}$ donnés par la procédure d'affinement présente un maximum autour de la direction $\langle 111\rangle$ du réseau cubique comme le montre la projection stéréographique de cette fonction (Fig. 5). D'autre part la convergence rapide de l'affinement $(l=6)$ indique une délocalisation importante de la molécule.

La détermination des coefficients $A_{l}^{1 \lambda^{\prime} \neq 1}$ donnant l'orientation uniaxiale de la molécule, soulève le problème signalé précédemment à savoir que le nombre de couches significatives (la couche de carbone en diffraction $\mathrm{X}$ ) qui prend en compte le désordre uniaxial est inférieur au nombre de représentations irréductibles $\lambda^{\prime}\left(l=6, \lambda^{\prime}=2\right)$. Dans ce cas les coefficients $A_{l}^{1 \lambda^{\prime}}$ doivent être contraints. Un calcul des facteurs de structure a été effectué pour différentes valeurs de $A_{6}^{1,2}$; le minimum du facteur de mérite nous fournit la valeur du coefficient (Fig. 6). Soit $A_{6}^{12} \# 0,35$.

Compte tenu de l'expression mathématique du rotateur $U_{6}^{12}$, il apparaît que cette valeur positive de $A_{6}^{12}$ donnent des maximums de probabilité uniaxiale à $\alpha=0^{\circ}$ et $\alpha=60^{\circ}$. Ce résultat est en accord avec le précédent résultat déduit du modèle de désordre statique.

D'une manière générale les résultats obtenus par les deux modèles sont comparables. Par exemple l'amplitude de libration de l'axe $\mathrm{C}_{3}$ moléculaire autour de sa direction d'équilibre donnée par $\left(L_{11}^{\mathrm{m}}\right)^{1 / 2}$ dans le premier modèle et que l'on peut extraire du second en ajustant la forme de la fonction $P_{0}\left(\alpha=45^{\circ}, \beta\right.$, $\gamma=0$ ) par une gaussienne (Fig. 7)

$$
P_{0}\left(\alpha=45^{\circ}, \beta, \gamma=0^{\circ}\right)=A \exp -\frac{\left(\beta-\beta_{\mathrm{e}}\right)^{2}}{2\left\langle\beta^{2}\right\rangle}
$$

où $A$ assure la norme et $\beta_{\mathrm{e}}=54^{\circ} 73$ est la position

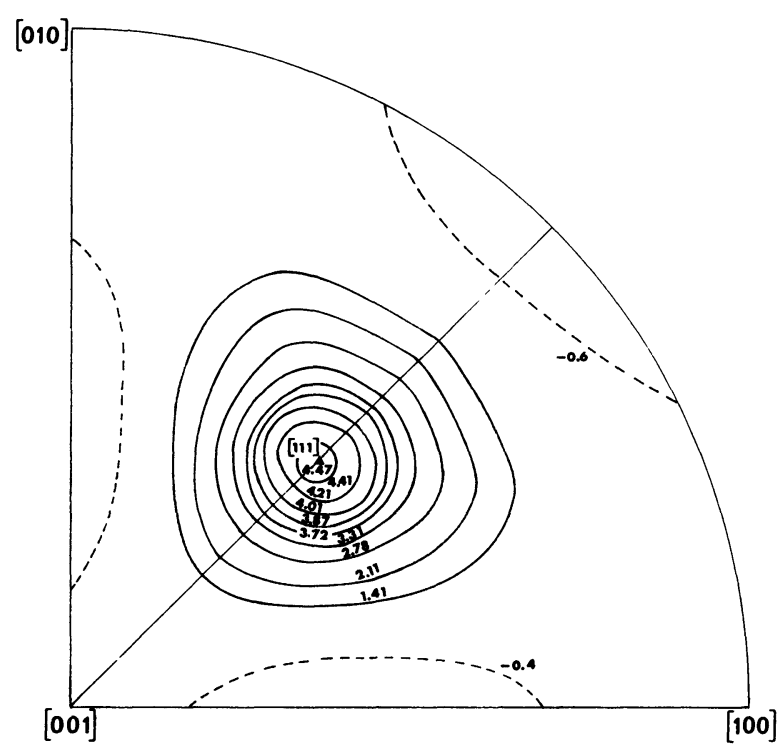

Fig. 5. - Projection stéréographique de la probabilité d'orientation $P(\Omega)$.

[Stereographic projection of the orientational molecular density.]

Tableau IV. - Paramètres structuraux de la triéthylènediamine relatifs au modèle utilisant les fonctions adaptées à la symétrie.

[Structural parameters of triethylenediamine corresponding to the model described by symmetry-adapted functions.]

$$
\begin{gathered}
A_{4}^{11} \\
-0,334 \\
(0,041)
\end{gathered}
$$

$\begin{array}{cc}A_{6}^{12} & \left\langle u_{x}^{2}\right\rangle\left(\AA^{2}\right) \\ \overline{0,35} & - \\ \text { (contraint) } & 0,166 \\ (0,010)\end{array}$

$(0,010)$

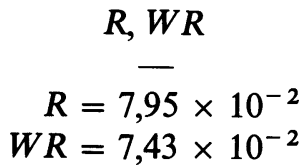




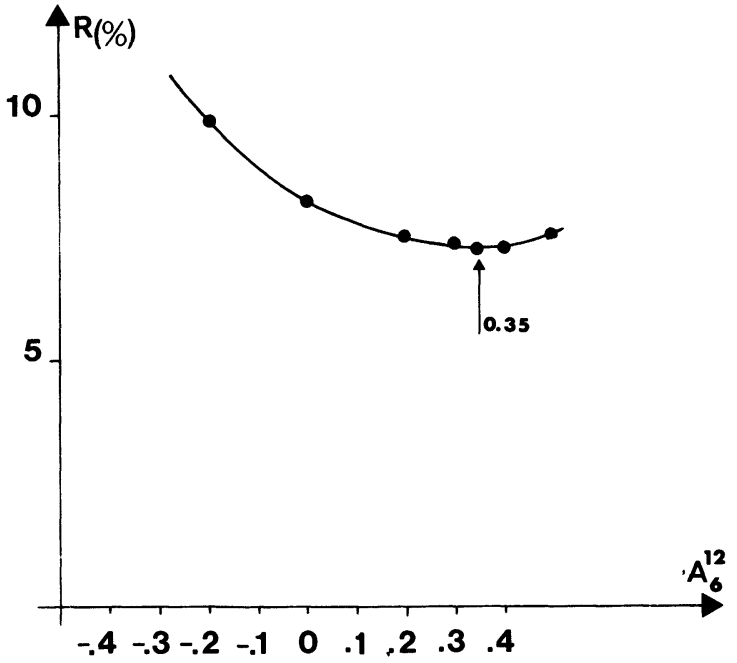

Fig. 6. - Variations du facteur de mérite $R$, pour différentes valeurs du coefficient $A_{6}^{12}$.

[Variations of the reliability factor $R$, for different values of the $A_{6}^{12}$ coefficient.]

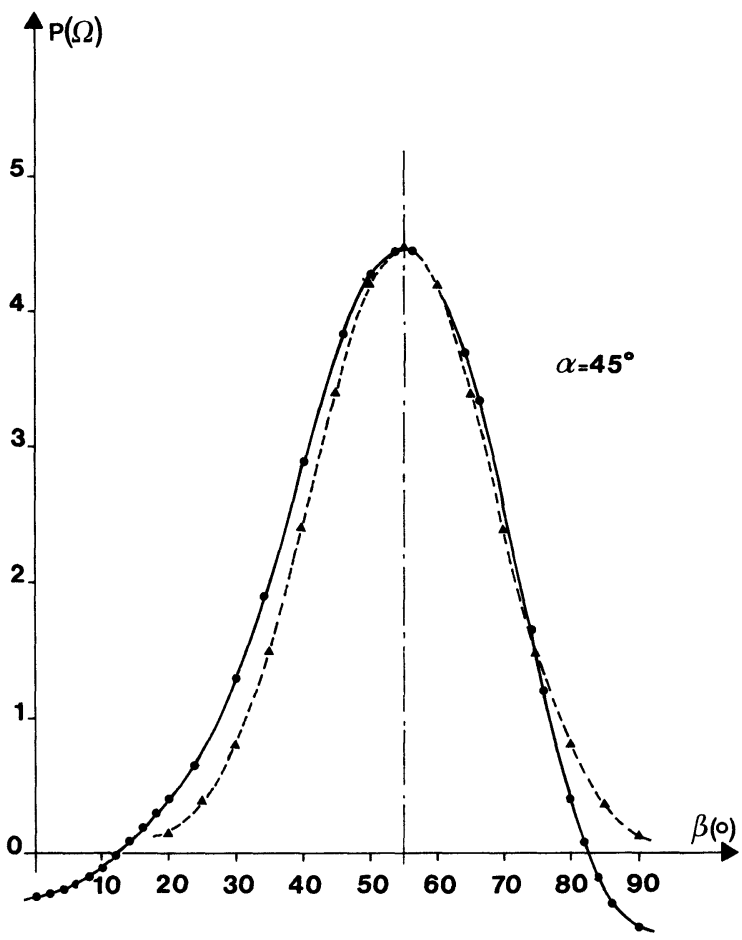

Fig. 7. - Représentations de la probabilité $P_{0}\left(\alpha=45^{\circ}, \beta\right.$, $\gamma=0^{\circ}$ ). Les symboles $(\bullet)$ et $(\Delta)$ correspondent respectivement à la probabilité issue de la procédure d'affinement et à son ajustement par la fonction $A \exp \left[-\frac{\left(\beta-\beta_{\mathrm{e}}\right)^{2}}{2\left\langle\beta^{2}\right\rangle}\right]$.

[Representations of the probability $P_{0}\left(\alpha=45^{\circ}, \beta, \gamma=0^{\circ}\right)$. Symbols $(\bullet)$ et $(\boldsymbol{\Delta})$ correspond respectively to the probability given by the refinement procedure and to its fit by the function $\left.A \exp \left[-\frac{\left(\beta-\beta_{\mathrm{e}}\right)^{2}}{2\left\langle\beta^{2}\right\rangle}\right]\right]$ moyenne de l'axe $\mathrm{C}_{3}$ moléculaire. On obtient :

$\left\langle\beta^{2}\right\rangle \# 0,050(\mathrm{rd})^{2}$ valeur cohérente avec

$$
L_{11}^{\mathrm{m}} \# 0,045(\mathrm{rd})^{2}
$$

la différence pouvant s'expliquer par le fait que $P_{0}(\Omega)$ n'est pas strictement décrite par une gaussienne.

La comparaison de ces résultats avec ceux relatifs aux composés de la même famille :

$$
\begin{aligned}
& \text { bicyclo (222) octane, }\left(\mathrm{BCO}, \mathrm{HC}\left(\mathrm{CH}_{2} \mathrm{CH}_{2}\right)_{3} \mathrm{CH}\right) \\
& \text { et } \\
& \text { quinuclidine, }\left(\mathrm{N}_{(}\left(\mathrm{CH}_{2} \mathrm{CH}_{2}\right)_{3} \mathrm{CH}\right)
\end{aligned}
$$

montrent que dans le cas de TEDA et de BCO qui sont des molécules de même symétrie $\left(D_{3 h}\right)$ les axes moléculaires d'ordre 3 s'alignent en moyenne le long des directions $\langle 111\rangle$ du cube avec une amplitude de libration d'environ $12^{\circ}$. La molécule possède alors 8 orientations d'équilibre discernables en chaque site. Dans le cas de la quinuclidine, la symétrie moléculaire est abaissée $\left(C_{3 v}\right)$ et l'axe $C_{3}$ moléculaire n'est plus le long d'une direction $\langle 111\rangle$, l'angle entre l'axe $C_{3}$ moléculaire et la direction $\langle 111\rangle$ la plus proche étant voisine de $10^{\circ} 5[10]$. Dans cette dernière analyse le nombre de réflexions significatives (47) est plus important que dans notre cas (TEDA : 17 raies). Il nous semble alors que l'ensemble de ces résultats est cohérent dans la mesure où notre conclusion suggère que dans le cas de TEDA l'axe $\mathrm{C}_{3}$ moléculaire se situe dans un cône de $12^{\circ}$ d'ouverture autour d'une direction $\langle 111\rangle$ sans que l'on soit capable de déterminer si cette distribution correspond ou non à une répartition d'orientations définies comme cela semble être le cas dans la quinuclidine.

\section{Conclusion.}

Les deux modèles étudiés conduisent à la description suivante du désordre d'orientation de la phase plastique de la triéthylènediamine. En accord avec Nimmo et Lucas l'axe d'ordre trois moléculaire est en moyenne le long des directions $\langle 111\rangle$ du cube, de même deux orientations uniaxiales de la molécule ont été trouvées. Toutefois les orientations uniaxiales trouvées par les deux modèles sont à $30^{\circ}$ de celles de Nimmo et Lucas. Nous tirons argument du fait que nous avons enregistré un plus grand nombre de raies de diffraction que Nimmo et Lucas et que nos mesures ont été effectuées sur un monocristal pour confirmer notre résultat. Il est à noter que les deux modèles qui ont des approches différentes, donnent sans ambiguité la même description de la structure. Ce résultat concernant ces positions d'équilibre a une conséquence importante sur le mécanisme de la transition ordredésordre (hcp-fcc) de la triéthylènediamine dans la mesure où il impose, contrairement aux positions de Nimmo et Lucas, une importante rotation des 

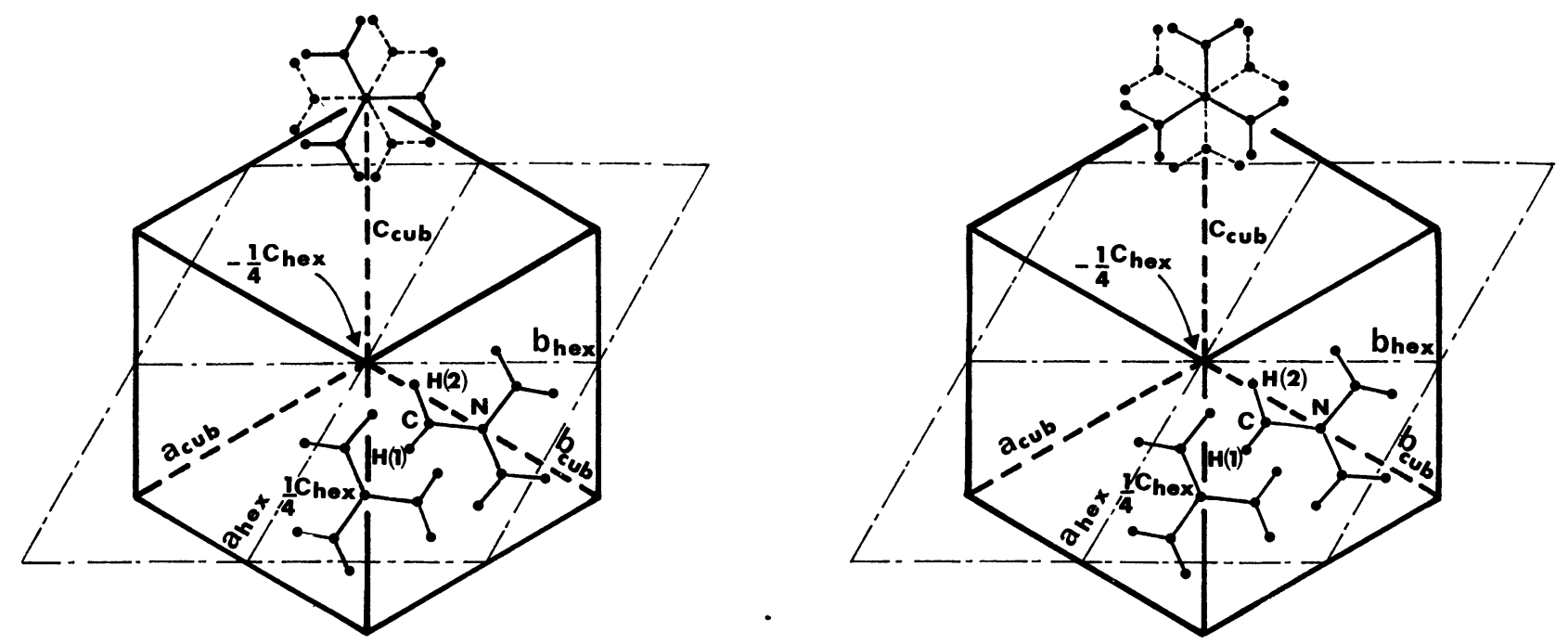

Fig. 8. - Relation entre les mailles hexagonale (hcp) et cubique (fcc). Le cube est vu le long de la direction $\langle 111\rangle$ suivant laquelle est orienté le vecteur $\mathrm{c}_{\text {hex }}$. Son centre est situé en $-\frac{1}{4} c_{\text {hex }}$. Les molécules représentées dans la partie inférieure droite de chaque figure sont dans leur position d'équilibre de la phase basse température. Les deux orientations possibles de la molécule autour de l'axe $\langle 111\rangle$ du cube sont représentées dans la partie supérieure de chaque figure. Celles représentées en (a) correspondent aux positions de Nimmo et Lucas et celles représentées en (b) correspondent à nos positions.

[The relation between the hexagonal (hcp) and fcc unit cells appropriate to phases II and I of TEDA respectively. The cube is viewed along its $\langle 111\rangle$ direction. Its centre is located at $-\frac{1}{4} c_{\text {hex }}$. The structure of phase II is illustrated at the lower right of the diagram. The two orientations of the molecule around the $\langle 111\rangle$ direction for phase I are shown. Those given in (a) correspond to the positions of Nimmo and Lucas and those given in (b) correspond to our positions.]

deux molécules de la phase basse température pour obtenir les orientations d'équilibre de la phase haute température [11] (Fig. 8).

De plus la transition de phase hcp $\rightarrow$ cfc est une transition martensitique. Perpendiculairement à un axe d'ordre 3 cristallographique on passe d'un empilement $\mathrm{ABAB}$... pour la phase hexagonale à un empilement $\mathrm{ABCABC}$... pour la phase cubique à faces centrées. Cette situation correspond à la transition à un important déplacement des centres de masses moléculaires couplé au désordre orientationnel. Cette transition est de ce fait fortement du $1^{\text {er }}$ ordre ce qui explique la destruction du cristal quand celle-ci a lieu.

\section{Bibliographie}

[1] Towbridge, J. C. et Westrum, E. V., J. Phys. Chem. 67 (1963) 2381-5.

[2] Nimmo, J. K. et LucAs, B. W., Acta Cryst. B 32 (1976) 348.

[3] Sauvajol, J. L., J. Phys. C 13 (1980) L927.

[4] SMith, G. W., J. Chem. Phys. 43 (1965) 4325-36.

[5] Nimmo, J. K. et LuCAS, B. W., Acta Cryst. B 32 (1976) 597.

[6] André, D., Fourme, R. et Renaud, M., Acta Cryst. B 27 (1971) 2371.
[7] Amoureux, J. P., Sauvajol, J. L., Bée, M., Acta Cryst. A 37 (1981) 97-104.

[8] Bradley, C. J. et Cracknell, A. P., The mathematical theory of symmetry in solids (Clarendon Press, Oxford) 1972.

[9] Sauvajol, J. L. et Amoureux, J. P., J. Phys. C : Solid State Phys. 14 (1981) 1537.

[10] Fourme, R., J. Physique 40 (1979) 67.

[11] Nimmo, J. K. et LuCAS, B. W., Acta Cryst. B 32 (1976) 2729. 